\title{
Cellular properties of nilpotent spaces
}

\author{
WOJCIECH CHACHÓLSKI \\ EMMANUEL DROR FARJOUN \\ RAMÓN FLORES \\ JÉRÔME SCHERER
}

\begin{abstract}
We show that cellular approximations of nilpotent Postnikov stages are always nilpotent Postnikov stages, in particular classifying spaces of nilpotent groups are turned into classifying spaces of nilpotent groups. We use a modified Bousfield-Kan homology completion tower $z_{k} X$ whose terms we prove are all $X$-cellular for any $X$. As straightforward consequences, we show that if $X$ is $\mathcal{K}$-acyclic and nilpotent for a given homology theory $\mathcal{K}$, then so are all its Postnikov sections $P_{n} X$, and that any nilpotent space for which the space of pointed self-maps $\operatorname{map}_{*}(X, X)$ is "canonically" discrete must be aspherical.
\end{abstract}

55P60, 20F18; 55N20, 55R35

\section{Introduction}

The Postnikov tower of a nilpotent space $X$ is considered classically as a way to approximate $X$ by inductively adding Eilenberg-Mac Lane spaces (basic homotopical building blocks) via principal fibration sequences; see Example 2.4 for what we mean by Postnikov sections. Eventually the space $X$ itself is recovered as homotopy limit of the Postnikov tower $P_{n} X$.

In this work we change the perspective and show that, if $X$ is nilpotent, then its Postnikov sections can themselves be constructed out of $X$ by means of wedges, homotopy pushouts and telescopes (see Theorem 6.6). This is certainly not true for arbitrary spaces and, when $X$ is nilpotent, it allows us to deduce strong properties of its Postnikov sections. Let us first mention a few of these consequences and then present our methods and techniques. We provide a topological strengthening of the classical Serre class statements on the relation between homotopy and homology groups; see Serre [25]. 
Theorem 7.11 Let $\mathcal{K}$ be a reduced homology theory.

(1) Assume $X$ is $\mathcal{K}$-acyclic. If $P_{n} X$ is nilpotent, then it is also $\mathcal{K}$-acyclic.

(2) Assume $X$ is nilpotent. Then $\prod_{k \geq 1} K\left(\pi_{k} X, k\right)$ is $\mathcal{K}$-acyclic if and only if $\prod_{k \geq 1} K\left(H_{k}(X, \mathbb{Z}), k\right)$ is $\mathcal{K}$-acyclic.

(3) If $G$ is a group such that $K(G, 1)$ is $\mathcal{K}$-acyclic, then so is $K\left(G / \Gamma_{n} G, 1\right)$ for any $n$.

In [2; 3, Lemma 5.3] Bousfield proved the "key lemma" as a step towards understanding the failure of preservation of fibrations by localization functors. This lemma implies for example that, if $X$ is a simply connected space, then the map $\pi_{n}: \operatorname{map}_{*}(X, X) \rightarrow$ $\operatorname{Hom}\left(\pi_{n} X, \pi_{n} X\right)$ is a weak equivalence if and only if $X$ is weakly equivalent to $K\left(\pi_{n} X, n\right)$. If $X$ is not simply connected, then this is far from being true. In this article we offer an extension of this last result to spaces with a nilpotent fundamental group.

Theorem 7.8 Let $X$ be a connected space whose fundamental group $\pi_{1} X$ is nilpotent. Assume that the map $\pi_{1}: \operatorname{map}_{*}(X, X) \rightarrow \operatorname{Hom}\left(\pi_{1} X, \pi_{1} X\right)$ is a weak equivalence. Then $X$ is weakly equivalent to $K\left(\pi_{1} X, 1\right)$.

For arbitrary fundamental groups, this fails as illustrated by the third and fourth authors in [20, Example 2.6] by a space $X$ with $\pi_{1} X \cong \Sigma_{3}$ whose universal cover is the homotopy fiber of the degree- 3 map on the sphere $S^{3}$. We come back to this space in Example 7.7.

To prove the above results we use cellularization techniques. Looking at spaces through the eyes of a given space $A$ via the pointed mapping $\operatorname{space}^{\operatorname{map}}(A,-)$ is the central idea; see the second author [16] and the first author [5]. Recently cellularization has found applications in other contexts: Dwyer, Greenlees and Iyengar used it to investigate duality in stable homotopy [14] (see also Shamir [26]); periodicity phenomena in unstable homotopy theory are the subject of the first author, Dwyer and Intermont [9]; cellularity has also been intensively studied in group theory (see for example the second author, Göbel and Segev [18] for an early general reference, and Blomgren, the first and second authors and Segev [1] for a recent point of view on finite (simple) groups); in [22] Kiessling computed explicitly the cellular lattice of certain perfect chain complexes, refining the Bousfield lattice.

Classically $A$ is the zero-sphere $S^{0}$ and we are doing standard homotopy theory. In this context the cellularization of a space is nothing else than the cellular approximation of the space, the best approximation built out of $S^{0}$ and its suspensions $S^{n}$ via pointed 
homotopy colimits. Changing the sphere for another space $A$ allows us to modify our point of view and we do now $A$-homotopy theory. The cellularization functor cell $_{A}:$ Spaces $_{*} \rightarrow$ Spaces $_{*}$ on pointed spaces retains from a space its essence from the point of view of $A$. A space of the form $\operatorname{cell}_{A} Y$ is $A$-cellular in the sense that it can be constructed from $A$ and its suspensions by pointed homotopy colimits. When a space $X$ is $A$-cellular we write $X \gg A$.

It turns out that to prove the above theorems we need to show that cell $A$ behaves nicely on Postnikov sections of any space $X$ provided that these sections are nilpotent. A result of the first author [5, Corollary 20.7] says that the structure map cell $A X \rightarrow X$ is a principal fibration. Hence, the cellularization of a nilpotent space is always nilpotent, which hints at the tame behavior of $\operatorname{cell}_{A}$ on nilpotent spaces. The tame behavior on nilpotent Postnikov sections is our main concern here.

We show in particular in Corollary 6.5 that if $X$ is a nilpotent $n$-Postnikov stage (see the end of Section 2), then so is $\operatorname{cell}_{A} X$. When $n=1$, this result is in perfect accord with a purely group-theoretical result, [18, Theorem 1.4(1)], showing that in the category of groups any cellularization of a nilpotent group is nilpotent. To understand how cellularization functors affect Postnikov sections, we rely on the following statement which we regard as the main result of this paper.

Theorem 6.6 Let $X$ be a space. If $P_{n} X$ is nilpotent, then $P_{n} X \gg X$, namely any nilpotent Postnikov section is $X$-cellular.

This theorem is a consequence of preservation of polyGEMs, as such, by cellularization functors. A space is called a 1-polyGEM if it is a GEM, ie a product of EilenbergMac Lane spaces $K\left(A_{i}, i\right)$ for abelian groups $A_{i}$. It is called an $n$-polyGEM, for $n>1$, if it is weakly equivalent to a retract of the homotopy fiber of a map $f: X \rightarrow Y$ where $X$ is an $(n-1)$-polyGEM and $Y$ is a GEM. We prove in Theorem 6.4 that cellularization functors turn polyGEMs into polyGEMs.

To prove the preservation of polyGEMs, namely that any cellularization of a polyGEM is again a polyGEM, we need detection tools for polyGEMs which behave well with respect to cellularity. It is well known that a space $X$ is a GEM if and only if it is a retract of $\mathbb{Z} X$, where $\mathbb{Z} X$ is the free abelian group construction on $X$ (see for example Curtis [12, Definition 3.5]) the simplicial version of the Dold-Thom infinite symmetric product $\mathrm{SP}^{\infty} X$ (see also Dold and Thom [13]). We need similar functors that detect the property of being a polyGEM. As a first attempt one might try to consider the functors $\mathbb{Z}_{k} X$ in the Bousfield-Kan completion tower with respect to the integral homology [4]. Although the spaces in this tower are polyGEMs, they are very special 
polyGEMs, so-called thin; see Libman [23]. Furthermore we do not know the needed cellularity properties of $\mathbb{Z}_{k} X$.

For our purposes, instead of the classical Bousfield-Kan completion tower, we consider the modified Bousfield-Kan tower $\left\{z_{k} X\right\}$ constructed by the second author in [17]. We do that in Section 5. The following two properties of this tower are essential in our work. First, it detects polyGEMs.

Proposition 5.5 A space $W$ is a polyGEM if and only if it is a retract of $z_{n} W$ for some $n$.

Second, the functors in the modified tower are cellular, a property that we are unable to prove for the functors in the classical Bousfield-Kan tower.

Proposition 5.6 For all $k \geq 0$ the coaugmented functor $z_{k}$ is cellular. In particular $z_{k} X$ is $X$-cellular for any $X$.

We define and study cellular functors in Section 4. The functor $z_{k}$ being cellular tells us much more than the fact that $z_{k} X$ is $X$-cellular. It says that the homotopy cofiber of the augmentation $X \rightarrow z_{k} X$ is $\Sigma X$-acyclic, so that $z_{k} X$ can be built from $X$ starting from $X$ and adding higher cells $\Sigma^{i} X$ for $i \geq 1$. This concept allows an inductive argument about $z_{k} X$ to proceed using a version of Bousfield's key lemma.

Acknowledgements We would like to thank the referee for helpful comments and Bill Dwyer for enlightening discussions and suggestions. W Chachólski was partially supported by Göran Gustafsson Stiftelse and VR grants. R Flores and J Scherer were partially supported by FEDER/MEC grant MTM2013-42293-P, and UNAB10-4E-378 "Una manera de hacer Europa". All authors thank the KTH in Stockholm for its hospitality.

\section{Notation and setup}

In this section we will recall basic definitions related to cellularity and acyclicity of spaces and state their fundamental properties. For more information about these notions we refer the reader to [16].

The category of pointed simplicial sets with the standard simplicial model structure is denoted by Spaces $_{*}$. Its objects are called pointed spaces or simply spaces, and morphisms are called maps. The space of maps between two pointed spaces $X$ and $Y$ is denoted by $\operatorname{map}_{*}(X, Y)$. 
We say that a class $\mathcal{C}$ of pointed spaces is closed under weak equivalences if, when $X$ belongs to $\mathcal{C}$, then so does any pointed space weakly equivalent to $X$. We say that $\mathcal{C}$ is closed under homotopy colimits if, for any functor $F: I \rightarrow$ Spaces $_{*}$ whose values belong to $\mathcal{C}$, the homotopy colimit hocolim $I F$ in Spaces $_{*}$ also belongs to $\mathcal{C}$. A class of pointed spaces $\mathcal{C}$ which is closed both under weak equivalences and homotopy colimits is called cellular.

Cellular classes were called closed classes in [16], but we believe that the name "cellular" is more descriptive in our context. For a class $\mathcal{C}$ to be cellular it is sufficient if it is closed under weak equivalences, arbitrary wedges and homotopy pushouts. This is so since all pointed homotopy colimits can be built by repeatedly using these two special cases. Furthermore, a retract of a member of a cellular class also belongs to the cellular class, [16, 2.D.1.5], where $X$ is a retract of $Y$ if there are maps $f: X \rightarrow Y$ and $r: Y \rightarrow X^{\prime}$ whose composition $r f$ is a weak equivalence.

The symbol $\mathcal{C}(A)$ denotes the smallest cellular class in Spaces $_{*}$ containing a given space $A$. If $X$ belongs to $\mathcal{C}(A)$, then we write $X \gg A$ and say that $X$ is $A$-cellular or that $A$ builds $X$. For example, $\mathcal{C}\left(S^{0}\right)$ consists of all pointed spaces and $\mathcal{C}\left(S^{n}\right)$ of all $(n-1)$-connected pointed spaces.

A weaker notion than cellularity is given by acyclicity (see [5] for the origin of the terminology). For a map $f: X \rightarrow Y$ of pointed spaces, Fib $(f)$ denotes the homotopy fiber of $f$ over the basepoint. A cellular class $\mathcal{C}$ is called acyclic if, for any map $f: X \rightarrow Y$ such that $Y$ and $\operatorname{Fib}(f)$ belong to $\mathcal{C}$, the space $X$ belongs to $\mathcal{C}$. We also say that $\mathcal{C}$ is closed under extensions by fibrations.

Given a pointed space $A$, the symbol $\overline{\mathcal{C}(A)}$ denotes the smallest acyclic class in Spaces $_{*}$ containing $A$. If a space $X$ belongs to $\overline{\mathcal{C}(A)}$, then we write $X>A$ and say that $X$ is $A$-acyclic. There is an obvious inclusion $\mathcal{C}(A) \subset \overline{\mathcal{C}(A)}$ which in general is proper. For example, if $p$ is a prime number and $G$ a finite $p$-group, $K(G, 1)$ is always $K(\mathbb{Z} / p, 1)$-acyclic; however, $K(G, 1)$ is $K(\mathbb{Z} / p, 1)$-cellular if and only if $G$ is generated by elements of order $p$; see the third author [19, Section 4] for details.

In [5], the first author proved that cellularity can be detected by means of a universal property:

Theorem 2.1 A pointed space $X$ is $A$-cellular if and only if, for any map $f$ between fibrant spaces such that $\operatorname{map}_{*}(A, f)$ is a weak equivalence, then $\operatorname{map}_{*}(X, f)$ is also a weak equivalence.

The analogous universal property for acyclicity is the following; see for example [5, Section 18]. 
Theorem 2.2 A space $X$ is $A$-acyclic if and only if, for any choice of basepoint in $A$ and $X$, and any fibrant space $Z$ such that $\operatorname{map}_{*}(A, Z)$ is contractible, then $\operatorname{map}_{*}(X, Z)$ is also contractible.

The present paper deals with possible values of the $A$-cellular approximation or $A-$ cellular cover of spaces. The existence and basic properties of these cellular cover functors cell $_{A}$ are guaranteed by the following result proved in [16, Section 2]; see also [5].

Theorem 2.3 Let $A$ be a pointed space.

(1) There is a natural fibration $c_{A, X}$ : $\operatorname{cell}_{A} X \rightarrow X$ in Spaces $_{*}$ such that:

- $\operatorname{cell}_{A}$ preserves weak equivalences.

- $\operatorname{cell}_{A} X$ is $A$-cellular.

- The map $\operatorname{map}_{*}\left(A, c_{A, X}\right)$ is a weak equivalence.

(2) A pointed space $X$ is $A$-cellular if and only if the map $c_{A, X}$ : $\operatorname{cell}_{A} X \rightarrow X$ is a weak equivalence.

The map $c_{A, X}$ : cell $A \rightarrow X$, given by Theorem 2.3, is called the $A$-cellular cover of $X$ and the functor cell $A$ : Spaces ${ }_{*} \rightarrow$ Spaces $_{*}$ the $A$-cellularization.

Example 2.4 Let $S^{n+1}$ be an $(n+1)$-dimensional sphere. The $S^{n+1}$-cellular cover $c_{S^{n+1}, X}:$ cell $_{S^{n+1}} X \rightarrow X$ coincides with the $n$-connected cover and fits into a fibration sequence

$$
\text { cell }_{S^{n+1}} X \stackrel{c_{S^{n+1}, X}}{\longrightarrow} X \stackrel{p_{n, X}}{\longrightarrow} P_{n} X,
$$

where $p_{n, X}: X \rightarrow P_{n} X$ is the $n^{\text {th }}$ Postnikov section. We call a space $X$ an $n-$ Postnikov stage if the map $p_{n, X}: X \rightarrow P_{n} X$ is a weak equivalence, that is, if $\pi_{i} X=0$ for $i \geq n+1$. Thus a 0 -Postnikov stage is homotopically discrete, and a connected 1 -Postnikov stage is an Eilenberg-Mac Lane space $K(\pi, 1)$.

Similarly to the $n$-connected cover, for any $A$, the map $c_{A, X}$ : cell $A \rightarrow X$ is always a principal fibration, [5, Corollary 20.7].

\section{Basic cellular inequalities}

This section contains fundamental cellular and acyclic inequalities. The idea is to use these inequalities as basic moves to obtain more involved ones. Although our basic result is an inequality $P_{n} X \gg X$ for a nilpotent $P_{n} X$, we will use on the way many 
general inequalities that hold without any nilpotency condition. The following results describe our basic dictionary of cellular inequalities, which in most cases admit direct proofs.

Our first statements, formulated for cellular inequalities $\gg$, hold also as stated for the weak inequality $>$. The weak, acyclic inequalities can be deduced from the cellular ones and the fact that, for any space $A$, there is a space $B$ such that $\overline{\mathcal{C}(A)}=\mathcal{C}(B)$; see the first author, Parent and Stanley [10, Corollary 6.2].

Proposition 3.1 Let $A$ and $X$ be pointed spaces.

(1) If $X \gg A$, then, for any space $E, E \wedge X \gg E \wedge A$.

(2) If $X$ is connected, then $\Omega X \gg A$ if and only if $X \gg S^{1} \wedge A$.

(3) If $X \gg A$ and $A$ is connected, then $\Omega X \gg \Omega A$.

(4) For any $X$ and $n \geq 1, \Omega^{n}\left(S^{n} \wedge X\right) \gg X$.

Proof The first cellular inequality follows from the universal property in Theorem 2.1 (see also the first author [7, Theorem 4.3]) while the corresponding acyclic universal property in Theorem 2.2 implies the weak inequality. The cellular inequality (2) is proved in [5, Theorem 10.8] and the acyclic one is [5, Theorem 18.5]. The inequalities (3) and (4) are easy consequences of (1) and (2).

Our second set of inequalities concerns homotopy fibers and homotopy cofibers of a map $f: X \rightarrow Y$. The last one tells us that the "fiber of the cofiber" is close to the space $X$ from a cellular point of view.

Proposition 3.2 Let $f: X \rightarrow Y$ be a map to a connected pointed space $Y$.

(5) $\operatorname{Cof}(f) \gg S^{1} \wedge \operatorname{Fib}(f)$.

(6) For any $E, \operatorname{Fib}(E \wedge f) \gg E \wedge \operatorname{Fib}(f)$.

(7) If $\alpha: Y \rightarrow \operatorname{Cof}(f)$ is a homotopy cofiber of $f$, then $\operatorname{Fib}(\alpha) \gg X$.

Proof Inequality (5) is [5, Proposition 10.5] while (6) follows from the case of a circle (see the second author [15]), and induction on a cell decomposition. Finally (7) is [16, Corollary 9.A.10] (see also [5, Proposition 4.5(4)]).

In fibration and cofibration sequences we can sometimes relate the cellularity type of the spaces involved in the sequence. The main difficulty for a fibration sequence $F \rightarrow E \rightarrow B$ is that in general we cannot extract information about the total space of the type $E \gg A$ knowing that $B, F \gg A$. 
Proposition 3.3 Let $Z \rightarrow X \rightarrow Y$ be either a cofibration or a fibration sequence.

(8) If $Z>A$ and $Y>A$, then $X>A$.

(9) If $Z \gg A$ and $Y>S^{1} \wedge A$, then $X \gg A$.

(10) If $Z>A$ and $X \gg A$, then $Y \gg A$.

Proof For a fibration sequence inequality (8) holds by definition of the relation $>$. The cofibration sequence case follows from (7) and the fibration sequence case. Inequality (9) is [5, Corollary 20.2] for a fibration sequence, and the cofibration case follows again from (7) and the fibration sequence case. Finally, inequality (10) may be deduced from statements (5) and (9).

The following inequalities, that put in relation fiber and cofiber from the cellular point of view, will be specially relevant in the rest of the paper.

Proposition 3.4 (11) Let $X$ be a connected pointed space and $n$ be a positive integer. Let $e_{n, X}: X \rightarrow \Omega^{n}\left(S^{n} \wedge X\right)$ be the adjoint to id: $S^{n} \wedge X \rightarrow S^{n} \wedge X$. Then $\operatorname{Fib}\left(e_{n, X}\right) \gg S^{1} \wedge \Omega X \wedge \Omega X$ and $\operatorname{Cof}\left(e_{n, X}\right) \gg X \wedge X$.

Proof If $n=1$, then the statement for the homotopy fiber is proved by the first author [6, Theorem 7.2]. We proceed by induction on $n$. Let $n>1$. The map $e_{n, X}$ factors as the composition

$$
X \rightleftharpoons \Omega^{n-1}\left(S^{n-1} \wedge X\right) \stackrel{\Omega^{n-1}\left(e_{1, S^{n-1} \wedge X}\right)}{\longrightarrow} \Omega^{n}\left(S^{n} \wedge X\right),
$$

which leads to a fibration sequence

$$
\operatorname{Fib}\left(e_{n-1, X}\right) \rightarrow \operatorname{Fib}\left(e_{n, X}\right) \rightarrow \operatorname{Fib}\left(\Omega^{n-1}\left(e_{1, S^{n-1} \wedge X}\right)\right)
$$

and a cofibration sequence

$$
\operatorname{Cof}\left(e_{n-1, X}\right) \rightarrow \operatorname{Cof}\left(e_{n, X}\right) \rightarrow \operatorname{Cof}\left(\Omega^{n-1}\left(e_{1, S^{n-1} \wedge X}\right)\right) .
$$

Let us analyze $\operatorname{Fib}\left(\Omega^{n-1}\left(e_{1, S^{n-1} \wedge X}\right)\right) \simeq \Omega^{n-1}\left(\operatorname{Fib}\left(e_{1, S^{n-1} \wedge X}\right)\right)$. According to statements (2) and (3) the following cellular relation holds:

$$
\Omega^{n-1}\left(S^{1} \wedge \Omega\left(S^{n-1} \wedge X\right) \wedge \Omega\left(S^{n-1} \wedge X\right)\right) \gg \Omega^{n-1}\left(S^{1} \wedge S^{n-2} \wedge X \wedge S^{n-2} \wedge X\right) .
$$

For this last space $\Omega^{n-1}\left(S^{2 n-3} \wedge X \wedge X\right)$, by the same argument, we have the relation $\Omega^{n-1}\left(S^{2 n-3} \wedge X \wedge X\right) \gg S^{n-2} \wedge X \wedge X \gg S^{n} \wedge \Omega X \wedge \Omega X \gg S^{2} \wedge \Omega X \wedge \Omega X$. 
Thus Fib $\left(e_{n, X}\right) \gg S^{1} \wedge \Omega X \wedge \Omega X$ is a consequence of the inductive step and statement (9). The case of the homotopy cofiber is an analogous induction based on the proof of [6, Theorem 7.2], which analyzes the James construction.

We arrive now at more delicate inequalities involving diagrams. Consider a homotopy pushout square:

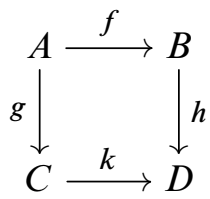

Let $\beta: \operatorname{Fib}(g) \rightarrow \operatorname{Fib}(h)$ and $\gamma: A \rightarrow T:=\operatorname{holim}(C \stackrel{k}{\rightarrow} D \stackrel{h}{\leftarrow} B)$ be the maps induced by the commutativity of this square.

Theorem 3.5 (12) If $C$ and $D$ are connected, then $\operatorname{Fib}(h) \gg \operatorname{Fib}(g)$.

(13) If $\operatorname{Fib}(k)$ is connected, then $\operatorname{Cof}(\beta) \gg S^{1} \wedge \operatorname{Fib}(g) \wedge \Omega \operatorname{Fib}(k)$.

(14) If $B, C, T$ and $\operatorname{Fib}(\gamma)$ are connected, then $\operatorname{Fib}(\gamma)>S^{1} \wedge \Omega \operatorname{Fib}(f) \wedge \Omega \operatorname{Fib}(g)$.

Proof Inequality (12) is [7, Theorem 3.4]. To check (13), observe that according to Puppe's theorem we can form the following homotopy-pushout square of homotopy fibers

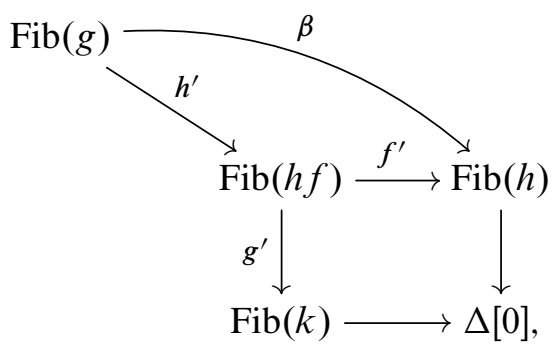

where the map $h^{\prime}: \operatorname{Fib}(g) \rightarrow \operatorname{Fib}(h f)$ is a homotopy fiber of $g^{\prime}: \operatorname{Fib}(h f) \rightarrow \operatorname{Fib}(k)$. Let $\operatorname{Fib}(k) \hookrightarrow R \operatorname{Fib}(k)$ be a weak equivalence into a fibrant space. Since the composition $g^{\prime} h^{\prime}$ factors through a contractible space, by taking the cofibers of $h^{\prime}$ and $\beta$, we can form a new homotopy pushout square

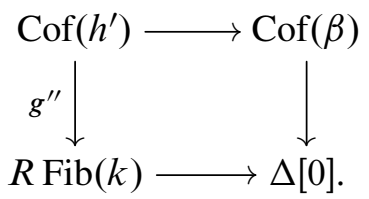

By Ganea's theorem [21], $\operatorname{Fib}\left(g^{\prime \prime}\right) \simeq S^{1} \wedge \operatorname{Fib}(g) \wedge \Omega \operatorname{Fib}(k)$. Under the assumption that $\operatorname{Fib}(k)$ is connected, we can then apply statement (13) to this last homotopy 
pushout square to get $\operatorname{Cof}(\beta) \gg S^{1} \wedge \operatorname{Fib}(g) \wedge \Omega \operatorname{Fib}(k)$. Finally, inequality (14) is by Werndli, the first and fourth authors [11, Theorem 5.1].

\section{Cellular functors and Bousfield key lemma}

An essential tool to understand the failure of preservation of fibrations by localizations and cellularizations is the so-called "key lemma" of Bousfield [3, Theorem 5.3] and the second author [16]. In its original form it states that if, for connected pointed spaces $X$ and $Y, \operatorname{map}_{*}(X, Y)$ is weakly equivalent to a discrete space and $\pi_{1} Y$ acts trivially on the set of components $\pi_{0} \operatorname{map}_{*}(X, Y)$, then the Hurewicz map $h_{X}: X \rightarrow \mathbb{Z} X$ induces a weak equivalence between $\operatorname{map}_{*}(X, Y)$ and $\operatorname{map}_{*}(\mathbb{Z} X, Y)$. The space $\mathbb{Z} X$ is the simplicial abelian group freely generated by the simplices of $X$ where the basepoint of $X$ is put equal to zero. This construction is left adjoint to the forgetful functor from simplicial abelian groups to pointed simplicial sets. It is the simplicial model for the Dold-Thom infinite symmetric product $\mathrm{SP}^{\infty} X \simeq \prod K\left(H_{n}(X ; \mathbb{Z}), n\right)$; see for example [12, 3.11].

The assumptions of the key lemma are equivalent to the statement that the inclusion $S^{1} \vee X \hookrightarrow S^{1} \times X$ induces a weak homotopy equivalence between the mapping spaces $\operatorname{map}_{*}\left(S^{1} \vee X, Y\right)$ and $\operatorname{map}_{*}\left(S^{1} \times X, Y\right)$ which means that $Y$ is local with respect to $S^{1} \vee X \hookrightarrow S^{1} \times X$. The key lemma states therefore that, for a connected space $X$, the

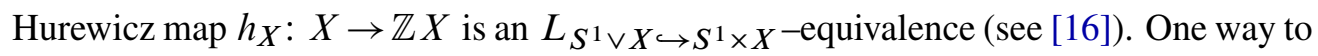
prove this lemma is to show a stronger statement: the map $h_{X}: X \rightarrow \mathbb{Z} X$ is constructed inductively starting with $X$ and taking pushouts along the inclusion $S^{1} \vee X \hookrightarrow S^{1} \times X$ or its suspensions. Since the cofiber of the map $S^{1} \vee X \hookrightarrow S^{1} \times X$ is $S^{1} \wedge X$, it follows that $\operatorname{Cof}\left(h_{X}\right)>S^{1} \wedge X$ (in fact a stronger relation $\operatorname{Cof}\left(h_{X}\right) \gg S^{1} \wedge X$ holds in this case) [16, Section 4.A]. Furthermore if $X$ is simply connected, the same argument can be used to show that the Hurewicz map $h_{X}: X \rightarrow \mathbb{Z} X$ is constructed inductively starting with $X$ and taking pushouts along the inclusion $S^{2} \vee X \hookrightarrow S^{2} \times X$ or its suspensions. By the Ganea theorem [21], Fib $\left(S^{2} \vee X \hookrightarrow S^{2} \times X\right) \simeq S^{1} \wedge \Omega S^{2} \wedge \Omega X$. Thus, if $X>S^{1} \wedge A$, for a connected $A$, then

$$
\operatorname{Fib}\left(S^{2} \vee X \hookrightarrow S^{2} \times X\right)>S^{1} \wedge S^{1} \wedge \Omega X>S^{1} \wedge S^{1} \wedge A=S^{2} \wedge A .
$$

We can then use Theorem 3.5(12) to infer that $\operatorname{Fib}\left(h_{X}\right)>S^{2} \wedge A$. These two properties of the Hurewicz map play an important role in this paper and therefore we are going to give them a name. Recall that a coaugmented functor $\mathcal{K}:$ Spaces $_{*} \rightarrow$ Spaces $_{*}$ is equipped by definition with a natural transformation $\mu_{X}: X \rightarrow \mathcal{K}(X)$ between the identity id: Spaces $_{*} \rightarrow$ Spaces $_{*}$ and $\mathcal{K}$. 
Definition 4.1 A coaugmented functor $\mu_{X}: X \rightarrow \mathcal{K}(X)$ is called cellular if:

(1) $\operatorname{Cof}\left(\mu_{X}: X \rightarrow \mathcal{K}(X)\right)>S^{1} \wedge X$ for any connected $X$.

(2) $\operatorname{Fib}\left(\mu_{X}: X \rightarrow \mathcal{K}(X)\right)>S^{2} \wedge A$ for any $X>S^{1} \wedge A$ and connected $A$.

The following is a consequence of Proposition 3.3(9).

Corollary 4.2 If $\mathcal{K}$ is a coaugmented cellular functor, then $\mathcal{K}(X)$ is $X$-cellular for any $X$.

Example 4.3 The natural unit map $X \rightarrow \Omega^{n}\left(S^{n} \wedge X\right)$ given by the "loop-suspension" adjunction defines a cellular functor, and so does the map $X \rightarrow Q X=\Omega^{\infty} \Sigma^{\infty} X$. Further examples can be constructed from connective ring spectra. If the unit map $S \rightarrow E$ induces an isomorphism on $\pi_{0}$, the functor $\Omega^{\infty}(E \wedge X)$ is cellular. The coaugmentation $X \rightarrow \mathrm{SP}^{n} X=X^{n} / \Sigma_{n}$ to the $n$-symmetric space satisfies requirement (1) of Definition 4.1 [16, Section 4.A], and one might ask if this map is also cellular. We believe it is, although we do not have a clear argument at the time of writing this paper.

Our main example of a coaugmented cellular functor is given by the Hurewicz map $h_{X}: X \rightarrow \mathbb{Z} X$. This follows from the discussion at the beginning of this section. We sum up the cellular properties of the functor $\mathbb{Z}$ in a proposition for future reference.

Proposition 4.4 The functor $\mathbb{Z}$ is cellular. In particular $\mathbb{Z} X \gg X$ for any space $X$. Moreover if $X>A$, then $\mathbb{Z} X>\mathbb{Z} A$.

Proof The second statement is Corollary 4.2. Finally we learn from [16, Proposition 7.B.5] that $P_{\mathbb{Z} A} \mathbb{Z} X=P_{A} \mathbb{Z} X$. Thus, if $A<X$, then $A<\mathbb{Z} X$ by the previous cellular inequality and so $P_{\mathbb{Z} A} \mathbb{Z} X$ is contractible, ie $\mathbb{Z} A<\mathbb{Z} X$.

How can we construct new coaugmented cellular functors out of old ones? For that we are going to use the following proposition. Let $f: X \rightarrow Y$ be a map. Take its homotopy cofiber $\alpha: Y \hookrightarrow \operatorname{Cof}(f)$ and the homotopy fiber $\operatorname{Fib}(\alpha) \rightarrow Y$ of $\alpha$. These maps fit into the commutative diagram

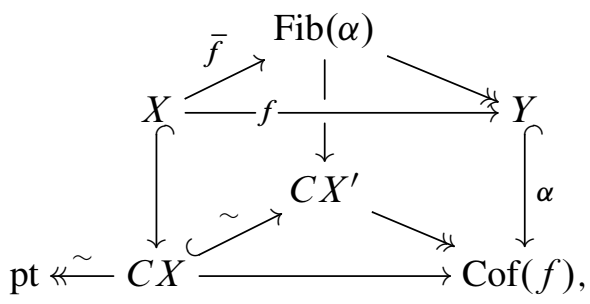


where the front square is a (homotopy) pushout, the right back square is a (homotopy) pullback and the indicated maps are weak equivalences, fibrations and cofibrations. The map $\bar{f}: X \rightarrow \operatorname{Fib}(\alpha)$ is called the comparison map.

Proposition 4.5 Let $f: X \rightarrow Y$ be a map, $\bar{f}$ as above, and $A$ be a connected space. (15) If $X$ is connected and $\operatorname{Cof}(f)$ is simply connected, then $\operatorname{Cof}(\bar{f}) \gg S^{1} \wedge X$. (16) If $\operatorname{Fib}(f)$ is simply connected and $X>S^{1} \wedge A$, then $\operatorname{Fib}(\bar{f})>S^{2} \wedge A$.

Proof We prove (15) first. By the assumption $\operatorname{Cof}(f)$ is 1 -connected. Thus its loop space, which is the homotopy fiber of $C X \rightarrow \operatorname{Cof}(f)$, is connected. We can therefore apply Theorem 3.5(13) to the homotopy pushout square

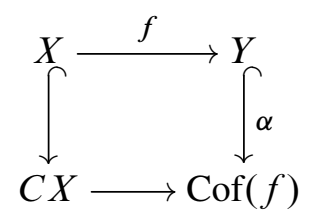

to get $\operatorname{Cof}(\bar{f}) \gg S^{1} \wedge X \wedge \Omega^{2} \operatorname{Cof}(f) \gg S^{1} \wedge X$.

To prove (16), assume $X>S^{1} \wedge A$. This implies $X$ is 1 -connected. According to Proposition 3.2(7) $\operatorname{Fib}(\alpha) \gg X$ and thus $\operatorname{Fib}(\alpha)$ is also 1-connected. The hypothesis of Theorem 3.5(14) is thus satisfied and we get $\operatorname{Fib}(\bar{f})>S^{1} \wedge \Omega X \wedge \Omega \operatorname{Fib}(f)$. Since $\mathrm{Fib}(f)$ is simply connected, its loop space $\Omega \mathrm{Fib}(f)$ is connected and we can conclude that $\operatorname{Fib}(\bar{f})>S^{1} \wedge \Omega X \wedge \Omega \operatorname{Fib}(f)>S^{2} \wedge \Omega X>S^{2} \wedge A$.

The cofiber-fiber construction applies to any coaugmented functor $\mathcal{K}$, taking first the homotopy cofiber of the coaugmentation $\mu_{X}: X \rightarrow \mathcal{K}(X)$ and then the homotopy fiber.

Definition 4.6 Let $\mathcal{K}$ be a coaugmented functor and $X$ any space. We denote by $\overline{\mathcal{K}}(X)$ the homotopy fiber of $\mathcal{K}(X) \hookrightarrow \operatorname{Cof}\left(\mu_{X}\right)$. The natural map $\bar{\mu}_{X}: X \rightarrow \overline{\mathcal{K}}(X)$ defines a new coaugmented functor $\overline{\mathcal{K}}$ : Spaces $_{*} \rightarrow$ Spaces $_{*}$.

Here is the main result of this section:

Proposition 4.7 Let $\mu_{X}: X \rightarrow \mathcal{K}(X)$ be a coaugmented functor. Assume that:

- If $X$ is connected, then $\operatorname{Cof}\left(\mu_{X}\right)$ is simply connected.

- If $X$ is simply connected, then so is $\mathcal{K}(X)$ and $\pi_{2}\left(\mu_{X}\right)$ is an epimorphism.

Then $\overline{\mathcal{K}}$ is a cellular coaugmented functor and thus $\overline{\mathcal{K}}(X)$ is $X$-cellular for any $X$. 
Proof Requirement (1) of Definition 4.1 follows from Proposition 4.5(15) and requirement (b) from Proposition 4.5(16). The fact that $\overline{\mathcal{K}}(X)$ is $X$-cellular is a direct consequence of Corollary 4.2.

For example the assumptions of Proposition 4.7 are satisfied if $\mathcal{K}$ is cellular:

Corollary 4.8 If $\mathcal{K}$ is a coaugmented cellular functor, then so is $\overline{\mathcal{K}}$.

\section{The modified Bousfield-Kan tower}

The aim of this section is to show that the coaugmented functors $\mu_{k, X}: X \rightarrow z_{k} X$ in a modified version of the integral Bousfield-Kan completion tower, as defined by the second author in [17], are cellular (see Definition 4.1). The modified tower was built originally as an elementary construction that models the pro-homology type of any space by a tower of much simpler spaces called polyGEMs.

Definition 5.1 A 1-polyGEM is defined to be a GEM, ie a space weakly equivalent to a product of abelian Eilenberg-Mac Lane spaces. For $n \geq 2$, an $n-p o l y G E M$ is a space which is weakly equivalent to a retract of the homotopy fiber of a map from an $(n-1)$-polyGEM to a GEM. A space is a polyGEM if it is an $n$-polyGEM for some integer $n$.

The above definition of $n$-polyGEM hides our ignorance of whether closing that class as we did under any retraction is necessary or not. PolyGEMs are examples of nilpotent spaces, and in a sense, universal such examples:

Proposition 5.2 A connected space $X$ is nilpotent if and only if, for any $n \geq 1$, the $n^{\text {th }}$ Postnikov section $P_{n} X$ is a polyGEM.

Proof If $X$ is nilpotent its Postnikov tower admits a refinement by principal fibrations whose fibers are Eilenberg-Mac Lane spaces. Conversely, if $P_{n} X$ is a polyGEM, it is nilpotent. Hence its fundamental group is nilpotent and acts nilpotently on all homotopy groups. This is true for all integers $n$, so $X$ itself is nilpotent.

How can we detect that a space is a polyGEM? This can be done using the integral Bousfield-Kan homology completion tower or, and this is the option we choose here, its modified version:

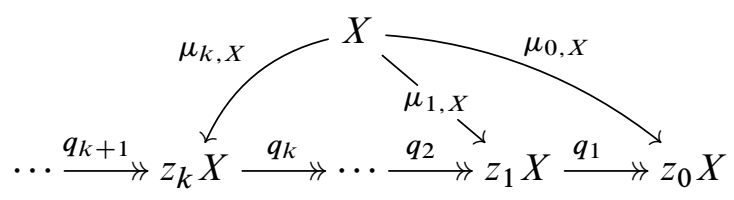


Recall from [17] the inductive construction of the tower. For $k=0, z_{0} X=\mathbb{Z} X$ and $\mu_{0, X}: X \rightarrow z_{0} X$ is the Hurewicz map $h_{X}: X \rightarrow \mathbb{Z} X$. For $k \geq 0$, the space $z_{k+1} X$ is the homotopy fiber of the composition

$$
z_{k} X \stackrel{\alpha}{\rightarrow} \operatorname{Cof}\left(\mu_{k, X}\right) \stackrel{h_{\operatorname{Cof}\left(\mu_{k, X}\right)}}{\longrightarrow} \mathbb{Z} \operatorname{Cof}\left(\mu_{k, X}\right),
$$

and the map $\mu_{k+1, X}: X \rightarrow z_{k+1} X$ fits into the following commutative diagram in which the horizontal sequences are homotopy fiber sequences:

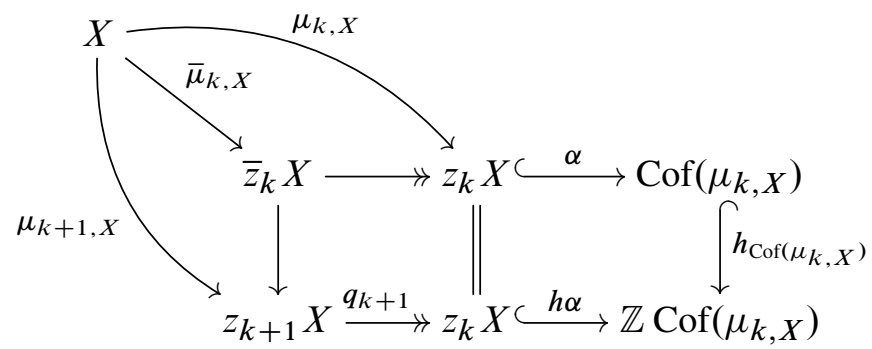

Observe that $z_{0} X$ is a GEM by definition, $z_{1} X$ is a 2-polyGEM as it is the homotopy fiber of a map between GEMs, and, more generally $z_{k} X$ is a $(k+1)$-polyGEM for any $k \geq 0$, as it is by induction the homotopy fiber of a map from a $k$-polyGEM to a GEM. Moreover, this new tower mimics the behavior of the classical Bousfield-Kan tower in the following sense:

Proposition 5.3 For any space $X$ the maps $\mu_{k, X}: X \rightarrow z_{k} X$, taken together as a map of towers, induce a pro-homology and ind-cohomology isomorphism between the constant tower $X$ and the modified integral Bousfield-Kan tower $\left(\cdots \rightarrow z_{1} X \rightarrow z_{0} X\right)$. Moreover, if $X$ is a polyGEM, then this map of towers induces a pro-isomorphism on pro-homotopy groups.

Proof The pro-homology isomorphism (and therefore also ind-cohomology isomorphism) holds by [17, Theorem 2.2]. As a corollary, see [17, Proposition 2.13], the map of towers induces a pro-isomorphism on pro-homotopy groups for any polyGEM, ie the kernel and cokernel are pro-isomorphic to zero. For more details see also the proof of Proposition 5.5.

Remark 5.4 A useful way to reformulate Proposition 5.3 is by saying that there is an ind-equivalence of towers of mapping spaces

$$
\operatorname{map}_{*}(X, K(A, n)) \rightarrow \operatorname{map}_{*}\left(z_{k} X, K(A, n)\right)
$$

for any abelian group $A$ and any natural number $n$. 
We can now state our detection criteria:

Proposition 5.5 A space $W$ is a polyGEM if and only if it is a retract of $z_{n} W$ for some $n$.

Proof (Compare with $[17,2.9]$ ) Since $z_{n} W$ is a polyGEM, by definition so is any of its retracts. That proves one implication.

Assume now that $W$ is a polyGEM. The maps $\mu_{k, W}: W \rightarrow z_{k} W$ induce an indcohomology equivalence as we just have seen in Proposition 5.3. Thus, for a fibrant GEM $P$, the maps of mapping spaces $\operatorname{map}_{*}\left(\mu_{k, W}, P\right): \operatorname{map}_{*}\left(z_{k} W, P\right) \rightarrow \operatorname{map}(W, P)$ induce an ind-homotopy equivalence. By induction, the same holds for any fibrant polyGEM $P$. To obtain the desired retraction, we can now use this ind-homotopy equivalence when $P$ is a fibrant replacement of $W$. The ind-isomorphism yields a map $z_{k} P \rightarrow P$ that corresponds to the identity $P \rightarrow P$, thus we get that $P$ is a retract of $z_{n} P$ for some natural number $n$.

We have only used in the above proof properties shared by the modified and the original Bousfield-Kan towers. The main result of the section, that is not known to hold for the original tower, is the following cellularity statement.

Proposition 5.6 For all $k \geq 0$, the coaugmented functor $z_{k}$ is cellular. In particular $z_{k} X$ is $X$-cellular for any $X$.

Proof The proof is by induction on $k$. The cellularity of $z_{0}=\mathbb{Z}$ was already discussed in Section 4; see Proposition 4.4. Assume the claim holds for some $k \geq 0$. Let us denote by $\beta: \bar{z}_{k} X \rightarrow z_{k+1} X$ the left vertical map in the diagram (*). This map fits into a commutative triangle

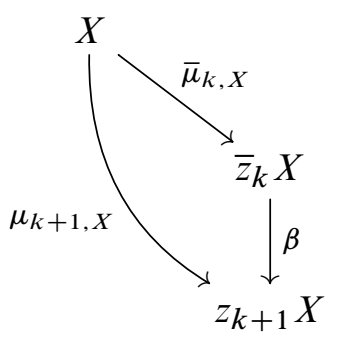

which exhibits $\mu_{k+1, X}$ as a composition of two maps, yielding both a cofibration and a fibration sequence:

$$
\operatorname{Cof}\left(\bar{\mu}_{k, X}\right) \rightarrow \operatorname{Cof}\left(\mu_{k+1, X}\right) \rightarrow \operatorname{Cof}(\beta), \quad \operatorname{Fib}\left(\bar{\mu}_{k, X}\right) \rightarrow \operatorname{Fib}\left(\mu_{k+1, X}\right) \rightarrow \operatorname{Fib}(\beta) .
$$


We will see in a moment that the sequence of three homotopy fibers above has a connected base space $\operatorname{Fib}(\beta)$, so it makes sense to speak about a fibration sequence since the homotopy fiber is well defined. We now use the cofibration sequence to prove part (1) of Definition 4.1 and then the above fibration sequence to prove part (2) of that definition of cellular functors.

Assume that $X$ is connected. Since the functor $z_{k}$ is cellular (by induction hypothesis), $\operatorname{Cof}\left(\mu_{k, X}\right)>S^{1} \wedge X$. We thus get the following inequality from the cellularity of the functor $\mathbb{Z}$ (part (2) of Definition 4.1):

$$
\Gamma \operatorname{Cof}\left(\mu_{k, X}\right):=\operatorname{Fib}\left(\operatorname{Cof}\left(\mu_{k, X}\right) \stackrel{h_{\operatorname{Cof}\left(\mu_{k, X}\right)}^{\longrightarrow}}{\longrightarrow} \operatorname{Cof}\left(\mu_{k, X}\right)\right)>S^{2} \wedge X .
$$

It follows that

$$
\operatorname{Fib}\left(\beta: \bar{z}_{k} X \rightarrow z_{k+1} X\right) \simeq \Omega \Gamma \operatorname{Cof}\left(\mu_{k, X}\right)>\Omega\left(S^{2} \wedge X\right)>S^{1} \wedge X .
$$

This implies that $\operatorname{Fib}(\beta)$ is $1-$ connected, hence connected as needed. In addition it follows that $\operatorname{Cof}(\beta)>S^{1} \wedge \operatorname{Fib}(\beta)>S^{2} \wedge X$ (see Proposition 3.3(8)). As $\bar{z}_{k}$ is cellular (see Corollary 4.8), we also have $\operatorname{Cof}\left(\bar{\mu}_{k, X}\right)>S^{1} \wedge X$. These last two inequalities imply $\operatorname{Cof}\left(\mu_{k+1, X}\right)>S^{1} \wedge X$ which is requirement (1) of Definition 4.1.

Assume now that $X>S^{1} \wedge A$ for a connected space $A$. Since $\bar{z}_{k}$ is a cellular functor, $\operatorname{Fib}\left(\bar{\mu}_{k, X}\right)>S^{2} \wedge A$. We have already seen that $\operatorname{Fib}(\beta)>S^{1} \wedge X$, and hence $\operatorname{Fib}(\beta)>S^{2} \wedge A$. These inequalities imply $\operatorname{Fib}\left(\mu_{k+1, X}\right)>S^{2} \wedge A$, which is requirement (2) of Definition 4.1. This concludes the induction step and the proof of the proposition.

We end this section with a useful lemma.

Lemma 5.7 Let $X$ be any connected space. For any $k \geq 0$ we have $\mathbb{Z} X<z_{k} X$.

Proof For $k=0$ there is nothing to prove. We proceed by induction. Assume that $k \geq 1$ and recall that, by definition, $z_{k} X$ fits into a fibration sequence:

$$
\Omega \mathbb{Z} \operatorname{Cof}\left(\mu_{k-1, X}\right) \rightarrow z_{k} X \rightarrow z_{k-1} X .
$$

As $z_{k-1}$ is cellular by Proposition 5.6, $\operatorname{Cof}\left(\mu_{k-1, X}\right)>S^{1} \wedge X$ and therefore we also have $\mathbb{Z} \operatorname{Cof}\left(\mu_{k-1, X}\right)>\mathbb{Z}\left(S^{1} \wedge X\right)$ by Proposition 4.4. This then implies that $\Omega \mathbb{Z} \operatorname{Cof}\left(\mu_{k-1, X}\right)>\mathbb{Z} X$. By induction we have $z_{k-1} X>\mathbb{Z} X$, and we can then conclude $z_{k} X>\mathbb{Z} X$. 


\section{Cellularity of Postnikov sections}

Recall that the Postnikov sections and highly connected covers are basic occurrences of nullifications and cellular covers: $P_{S^{n+1}} X$ is the $n^{\text {th }}$ Postnikov section $P_{n} X$ and cell $_{S^{n+1}} X$ is the $n$-connected cover. We turn to the proof of the main theorem of this article. Consider an arbitrary, not necessarily nilpotent space. Of course, a priori not a single Postnikov section need be nilpotent, but if there are some, they are built by the space we started with. This is in contrast with the situation for higher connected covers, as the following example illustrates.

Example 6.1 In general, an $n$-connected cover cell $S_{S^{n+1}} X$-even of a nilpotent space $X$-is not $X$-cellular. Consider for example $K(\mathbb{Z}, 2) \vee K(\mathbb{Z}, 2)$ whose cellular class is that of $K(\mathbb{Z}, 2)$. Its 2-connected cover is $S^{3}$ and the 3 -sphere is not $K(\mathbb{Z}, 2)$-cellular, in fact not $K(\mathbb{Z}, 2)$-acyclic. To see this let $M(\mathbb{Z} / 2,3)$ be the double suspension of $\mathbb{R} P^{2}$. This is a finite complex so, by the Sullivan conjecture (see Miller [24]),

$$
\operatorname{map}_{*}(K(\mathbb{Q} / \mathbb{Z}, 1), M(\mathbb{Z} / 2,3)) \simeq * .
$$

The space $K(\mathbb{Q} / \mathbb{Z}, 1)$ is the homotopy fiber of the map $K(\mathbb{Z}, 2) \rightarrow K(\mathbb{Q}, 2)$ induced by the inclusion $\mathbb{Z} \subset \mathbb{Q}$. By the above consequence of the Sullivan conjecture we then have

$$
\operatorname{map}_{*}(K(\mathbb{Z}, 2), M(\mathbb{Z} / 2,3)) \simeq \operatorname{map}_{*}(K(\mathbb{Q}, 2), M(\mathbb{Z} / 2,3)) .
$$

However, since $K(\mathbb{Q}, 2)$ is a rational space, $\operatorname{map}_{*}(K(\mathbb{Q}, 2), M(\mathbb{Z} / 2,3))$ is contractible and consequently so is $\operatorname{map}_{*}(K(\mathbb{Z}, 2), M(\mathbb{Z} / 2,3))$. This shows that $M(\mathbb{Z} / 2,3)$ can not be $K(\mathbb{Z}, 2)$-acyclic since this equation implies that its $K(\mathbb{Z}, 2)$-nullification is equivalent to itself, and thus cannot be contractible as required by the definition of $A$-acyclic space. As $M(\mathbb{Z} / 2,3)>S^{3}, S^{3}$ can not be $K(\mathbb{Z}, 2)$-acyclic either.

The following cellular and acyclic properties of polyGEMs are central to the proof of our main result. In Example 6.1 we have seen that in general the $n$-connected cover cell $_{S^{n+1}} X$ of a nilpotent space $X$ can fail to be $X$-acyclic. This however can not happen when $X$ is a polyGEM:

Proposition 6.2 Let $W$ be a polyGEM. Then for any $n \geq 0$ :

(1) $\operatorname{cell}_{S^{n}} W>W$.

(2) $K\left(\pi_{n} W, n\right)>W$.

(3) $P_{n} W \gg W$ and in particular $K\left(\pi_{1} W, 1\right) \gg W$.

(4) $\overline{\mathcal{C}(W)}=\overline{\mathcal{C}(\mathbb{Z} W)}=\overline{\mathcal{C}\left(\prod_{k \geq 0} K\left(\pi_{k} W, k\right)\right)}$. 
We first prove a version of Proposition 6.2(1) above for very special polyGEMs:

Lemma 6.3 Let $X$ be a space. For any $k \geq 0$ and $n \geq 0$, $\operatorname{cell}_{S^{n}} z_{k} X>X$.

Proof If $X$ is not connected, then the lemma is clear as all spaces are $X$-acyclic. Assume $X$ is connected. The proof is by induction on $k$. For $k=0$, the space $z_{0} X=\mathbb{Z} X$ is a GEM. Thus for any $n \geq 0$, cell $S^{n} \mathbb{Z} X$ is a retract of $\mathbb{Z} X$ and since $\mathbb{Z} X$ is $X$-cellular, then so is cell $_{S^{n}} \mathbb{Z} X$.

Take now $k>0$. Since $X$ is connected, then so is $z_{k} X$ and hence cell $S_{S^{0}} z_{k} X$ and cell $_{S^{1}} z_{k} X$ are weakly equivalent to $z_{k} X$, which is $X$-acyclic (even cellular) by Proposition 5.6. Suppose $n \geq 2$ and form the following commutative diagram where the horizontal sequences are fibration sequences and both the left and right vertical maps are (the usual higher connected) cellular covers (see [16, Theorem E2]):

$(* *)$

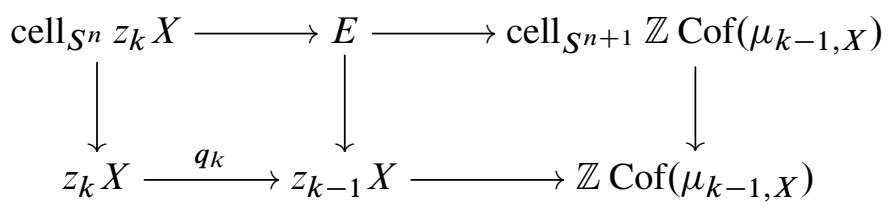

Explicitly, the space $E$ is the $(n-1)$-connected cover of the homotopy pullback of the right hand side pullback diagram. Since $z_{k-1}$ is a cellular functor (see Proposition 5.6), $\operatorname{Cof}\left(\mu_{k-1, X}\right)>S^{1} \wedge X$. This, together with the case $k=0$, gives

$$
\operatorname{cell}_{S^{n+1}} \mathbb{Z} \operatorname{Cof}\left(\mu_{k-1, X}\right)>\operatorname{Cof}\left(\mu_{k-1, X}\right)>S^{1} \wedge X
$$

or equivalently, by Proposition 3.1(2), $\Omega$ cell $_{S^{n+1}} \mathbb{Z} \operatorname{Cof}\left(\mu_{k-1, X}\right)>X$. To show cell $_{S^{n}} z_{k} X>X$ it is therefore enough to prove $E>X$ (see Proposition 3.3(8)).

The space $E$ is $(n-1)$-connected and the map $E \rightarrow z_{k-1} X$ induces an isomorphism on homotopy groups $\pi_{i}$ for $i \geq n+1$. We have thus a fibration sequence

$$
\operatorname{cell}_{S^{n+1}} z_{k-1} X \rightarrow E \rightarrow K\left(\pi_{n} E, n\right) .
$$

By the inductive assumption cell $S^{n+1} z_{k-1} X>X$. The inequality $E>X$ will then follow once we show $K\left(\pi_{n} E, n\right)>X$.

Let $G=\pi_{n} z_{k-1} X, H=\pi_{n} \mathbb{Z} \operatorname{Cof}\left(\mu_{k-1, X}\right)$ and $f: G \rightarrow H$ be the group homomorphism induced on $\pi_{n}$ by the map $z_{k-1}(X) \rightarrow \mathbb{Z} \operatorname{Cof}\left(\mu_{k-1, X}\right)$. By the inductive assumption cell $_{S^{n}} z_{k-1} X>X$ and $\operatorname{cell}_{S^{n+1}} z_{k-1} X>X$. These spaces fit into the fibration sequence

$$
\text { cell }_{S^{n+1}} z_{k-1} X \rightarrow \operatorname{cell}_{S^{n}} z_{k-1} X \rightarrow K(G, n) .
$$


It follows that $K(G, n)>X$. As $K(H, n)$ is a retract of $\mathbb{Z} \operatorname{Cof}\left(\mu_{k-1, X}\right)$, we also have $K(H, n)>\operatorname{Cof}\left(\mu_{k-1, X}\right)>S^{1} \wedge X$. These inequalities imply

$$
K(\operatorname{Ker}(f), n) \times K(\operatorname{Coker}(f), n-1) \simeq \operatorname{Fib}(K(f, n): K(G, n) \rightarrow K(H, n))>X .
$$

Hence, as a retract of $\operatorname{Fib}(K(f, n))$, the space $K(\operatorname{Ker}(f), n)$ is also $X$-acyclic. The long exact sequences in homotopy for the fibrations in the above diagram $(* *)$ allow us to identify $\pi_{n} E$ with $\operatorname{Ker}(f: G \rightarrow H)$. We conclude that $K\left(\pi_{n} E, n\right)>X$.

Proof of Proposition 6.2 If $W$ is not connected, then all four statements are clear. Assume then that $W$ is connected.

(1) Since $W$ is a polyGEM, Proposition 5.5 implies that it is a retract of $z_{k} W$ for some $k$. By functoriality, cell $S^{n} W$ is then a retract of $\operatorname{cell}_{S^{n}} z_{k} W$ and we conclude by Lemma 6.3 that cell $_{S^{n}} W>W$.

(2) This is a consequence of (1) and the fact that we have a fibration sequence

$$
\operatorname{cell}_{S^{n+1}} W \rightarrow \operatorname{cell}_{S^{n}} W \rightarrow K\left(\pi_{n} W, n\right) .
$$

(3) For $n=0$ the result is immediate as $P_{0} W$ is a retract of $W$. Let $n \geq 1$. In this case the statement follows from (1) and Proposition 3.3(10) applied to the fibration sequence cell $S^{n+1} W \rightarrow W \rightarrow P_{n} W$.

(4) We proved in Lemma 5.7 that $z_{k} W>\mathbb{Z} W$. Moreover, since $W$ is a polyGEM, there is an integer $k$ for which $W$ is a retract of $z_{k} W$. For such a $k$ we then have the relations $W \gg z_{k} W>\mathbb{Z} W \gg W$ which proves the equality $\overline{\mathcal{C}(W)}=\overline{\mathcal{C}(\mathbb{Z} W)}$. The inequality $\left.\prod K\left(\pi_{k} W, k\right)\right)>W$ follows from statement (2) since the infinite product is a pointed homotopy colimit of the finite ones. To conclude that the acyclic classes $\overline{\mathcal{C}(W)}$ and $\overline{\mathcal{C}\left(\prod K\left(\pi_{k} W, k\right)\right)}$ coincide what remains is the proof of the relation $\mathbb{Z} W>\prod K\left(\pi_{k} W, k\right)$. For any $n \geq 0$, the inequality $K\left(H_{n}(W), n\right) \gg \mathbb{Z}\left(P_{n} W\right)$ holds since $W$ and $P_{n} W$ have isomorphic $n^{\text {th }}$ integral homology groups by the Whitehead theorem. Therefore

$$
K\left(H_{n}(W), n\right) \gg \mathbb{Z}\left(P_{n} W\right) \gg P_{n} W>\prod_{k \geq 0} K\left(\pi_{k} W, k\right) .
$$

This implies that $\mathbb{Z} W>\prod_{k \geq 0} K\left(\pi_{k} W, k\right)$.

Before proving our main cellular inequality we start with the preservation of polyGEMs by general cellularizations. These are direct consequences of Proposition 6.2.

Theorem 6.4 If $X$ is a polyGEM, then so is $\operatorname{cell}_{A} X$ for any space $A$. 
Proof Assume $X$ is a polyGEM. It is thus a retract of $z_{n} X$ (see Proposition 5.5), for some $n$, ie there is a homotopy retraction $r$ of $\mu_{n, X}$ to a space $Y$ weakly equivalent to $X$. Hence we can form the (undotted part of the) following diagram with the indicated maps being weak equivalences and which, with the dotted arrow removed, is commutative by the functorial nature of the constructions:

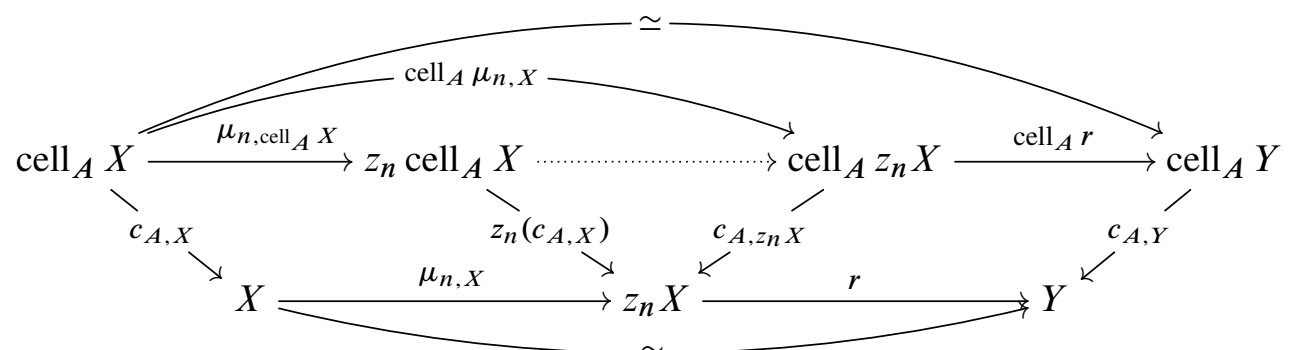

To construct the dotted arrow, note that since $z_{n}$ is a cellular functor (see Proposition 5.6), $z_{n}$ cell $_{A} X \gg \operatorname{cell}_{A} X \gg A$. We can then use the universal property of the $A$-cellular cover $c_{A, z_{n} X}$ to obtain the existence of the dotted arrow that makes the entire diagram homotopy commutative. Commutativity of this diagram shows that $\operatorname{cell}_{A} X$ is a retract of $z_{n} \operatorname{cell}_{A} X$ and hence by Proposition $5.5 \operatorname{cell}_{A} X$ is a polyGEM.

An analogous result to Theorem 6.4 holds also for finite nilpotent Postnikov stages.

Corollary 6.5 If $X$ is a nilpotent $n-P o s t n i k o v$ stage, then so is cell $A$ X for any $A$.

Proof Assume $p_{n, X}: X \rightarrow P_{n} X$ is a weak equivalence and $P_{n} X$ is nilpotent. Consider the following (solid arrows) diagram where the indicated maps are weak equivalences and, with the dotted arrow removed, it is commutative by functoriality of the constructions:

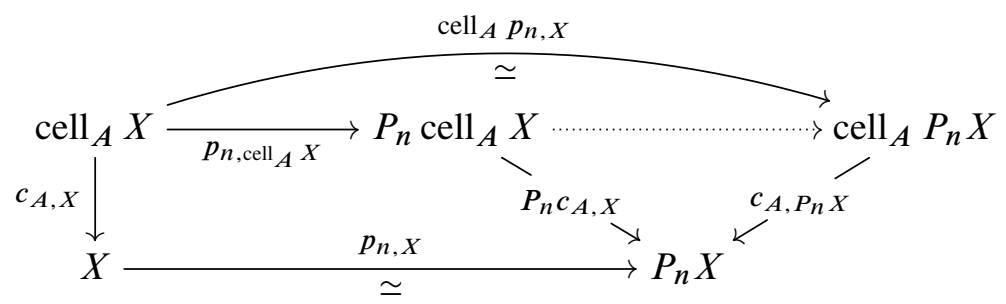

Since $X$ is a polyGEM (see Proposition 5.2), then so is $\operatorname{cell}_{A} X$ by Theorem 6.4. We can then use Proposition 6.2(3) to conclude that $P_{n}$ cell $_{A} X \gg \operatorname{cell}_{A} X \gg A$. The universal property of the cellular cover $c_{A}, P_{n} X$ gives the existence of the dotted arrow making the above diagram homotopy commutative. The map $p_{n, X}$ is an equivalence by assumption and thus so is the curved arrow. This implies that cell ${ }_{A} X$ is a homotopy retract of a Postnikov stage and hence is itself an $n$-Postnikov stage as claimed. 
We now turn to more general spaces. The following is our main new cellular inequality and in a sense the most easily stated result of the present work:

Theorem 6.6 Let $X$ be a space. If $P_{n} X$ is nilpotent, then $P_{n} X \gg X$.

Proof If $X$ is not connected, then the conclusion is clear. So assume $X$ is connected and $P_{n} X$ is nilpotent. The map $X \rightarrow P_{n} X$ factors through cell $X P_{n} X$ since $X$ is $X$-cellular, but we have seen in Corollary 6.5 that cell ${ }_{X} P_{n} X$ is an $n$-Postnikov stage. Thus the map $X \rightarrow \operatorname{cell}_{X} P_{n} X$ factors through $P_{n} X$, which implies that $P_{n} X$ is a retract up to homotopy of an $X$-cellular space, and hence it is $X$-cellular.

\section{Applications}

In this section we state various consequences of Proposition 5.6 and Theorem 6.6. We start with results related to polyGEMs and Postnikov sections. Theorem 6.4 can be used to give a description of all cellular covers of the classifying space of a nilpotent group in terms of the group-theoretical covers, see $[1 ; 18]$, and the first and second authors, Damian and Segev [8]. In general of course, cell ${ }_{A} K(G, 1)$ can have rich higher homotopy groups, eg the stable homotopy groups of spheres.

Proposition 7.1 Assume that $G$ is a nilpotent group and $A$ a connected space. Then $\operatorname{cell}_{A} K(G, 1) \simeq K\left(\operatorname{cell}_{\pi_{1} A} G, 1\right)$, where cell $\pi_{\pi_{1} A} G$ is the group theoretical $\pi_{1} A-$ cellularization of $G$ (see $[1 ; 8 ; 18])$.

Proof Corollary 6.5 implies that cell $_{A} K(G, 1) \simeq K(H, 1)$, where $H$ is a nilpotent group. Furthermore we claim that since $K(H, 1)$ is $A$-cellular, the group $H$ must be $\pi_{1} A$-cellular. To see this note that, by the Seifert-van Kampen theorem, the collection of all connected spaces with $\pi_{1} A$-cellular fundamental group is a cellular class. Since it contains $A$, it has to include the smallest cellular collection $\mathcal{C}(A)$, and in particular it contains $K(H, 1)$.

By the universal property of the cellularization and Theorem 2.3(1), the following map is a weak equivalence:

$$
\operatorname{map}_{*}\left(A, c_{A, K(G, 1)}\right): \operatorname{map}_{*}(A, K(H, 1)) \rightarrow \operatorname{map}_{*}(A, K(G, 1)) .
$$

Thus, on $\pi_{1}$, we get that the homomorphism $\pi_{1} c_{A, K(G, 1)}: H \rightarrow G$ induces a bijection

$$
\operatorname{Hom}\left(\pi_{1} A, \pi_{1} c_{A, K(G, 1)}\right): \operatorname{Hom}\left(\pi_{1} A, H\right) \cong \operatorname{Hom}\left(\pi_{1} A, G\right) .
$$

This homomorphism $\pi_{1} c_{A, K(G, 1)}: H \rightarrow G$ is therefore the $\pi_{1} A$-cellularization and $H$ is isomorphic to $\operatorname{cell}_{\pi_{1} A} G$. 
By [18, Theorem 1.4 (2)], the group-theoretical cellularizations of a finite nilpotent group $N$ are always subgroups of $N$.

Corollary 7.2 Let $P$ be a finite $p$-group. Then $\operatorname{cell}_{A} K(P, 1)$ is a $K\left(P^{\prime}, 1\right)$ for some subgroup $P^{\prime}$ of $P$.

It is sometimes possible to compute all possible such cellularizations.

Example 7.3 Let $D_{2^{n}}$ denote the dihedral group of order $2^{n}$ for $n \geq 2$. This is the group of symmetries of a regular polygon with $2^{n-1}$ sides and it is nilpotent of class $n-1$. The third author showed in [19, Proposition 5.1] that $D_{2^{n}}$ is $\mathbb{Z} / 2-$ cellular. We hence have only two possible cellularizations: cell $_{A} K\left(D_{2^{n}}, 1\right)$ can be contractible or cell $_{A} K\left(D_{2^{n}}, 1\right) \simeq K\left(D_{2^{n}}, 1\right)$. The latter is obtained for example for $A=K(\mathbb{Z} / 2,1)$. For $n \leq 3$, we were able to perform these computations by hand, but already for $n=4$ we do not know of a direct calculation of all cellularizations of $K\left(D_{16}, 1\right)$ without showing first that they must be $K(G, 1)$ 's.

The following is a particular case of Theorem 6.6, for $n=1$. Examples in which this result holds motivated the present work.

Corollary 7.4 If $\pi_{1}(X)$ is nilpotent, then $K\left(\pi_{1} X, 1\right) \gg X$.

We can also use Theorem 6.6 to get a Serre class-type statement that describes a global relation between the integral homology groups and the homotopy groups of a nilpotent space. No spectral sequence is needed in our proof, even though it seems that one could also obtain the mutual acyclicity of the homotopy and homology groups by a spectral sequence argument.

Corollary 7.5 If $X$ is nilpotent, then $\overline{\mathcal{C}(\mathbb{Z}(X))}=\overline{\mathcal{C}\left(\prod_{k \geq 0} K\left(\pi_{k} X, k\right)\right)}$.

Proof If $X$ is not connected, the statement is clear. Thus assume $X$ is connected. Even without the nilpotency assumption on $X$, for any $n \geq 0$, we have

$$
K\left(H_{n}(X), n\right)=K\left(H_{n}\left(P_{n} X\right), n\right) \gg \mathbb{Z}\left(P_{n} X\right) \gg P_{n} X>\prod_{k \geq 0} K\left(\pi_{k} X, k\right) .
$$

Consequently $\mathbb{Z}(X)>\prod_{k \geq 0} K\left(\pi_{k} X, k\right)$. For the opposite inequality, we need the assumption that $X$ is nilpotent, which, according to Proposition 5.2, is equivalent to $P_{n} X$ being a polyGEM for any $n \geq 0$. We can then use Theorem 6.6 and 
Proposition 6.2(2) to obtain $K\left(\pi_{n} X, n\right)>P_{n} X \gg X$ which, for $n>1$, implies $K\left(\pi_{n} X, n\right) \gg \mathbb{Z}\left(K\left(\pi_{n} X, n\right)\right)>\mathbb{Z}(X)$, where the last inequality is Proposition 4.4. For $n=1$, since $\pi_{1} X$ is nilpotent, we also have $K\left(\pi_{1} X, 1\right)>\mathbb{Z}(X)$. This shows $\prod_{k \geq 0} K\left(\pi_{k} X, k\right)>\mathbb{Z}(X)$.

We can also strengthen Corollary 7.4. Instead of assuming that the fundamental group of $X$ is nilpotent we make it nilpotent by taking the quotient by some stage of the lower central series.

Corollary 7.6 For any space $X$ and any $n \geq 1, K\left(\pi_{1} X / \Gamma_{n} \pi_{1} X, 1\right) \gg X$.

Proof Let $G$ be the fundamental group $\pi_{1} X$. If $X$ is not connected, the corollary is clear. Assume $X$ is connected. In this case according to Proposition 7.1, $\operatorname{cell}_{X} K\left(G / \Gamma_{n} G, 1\right) \simeq K\left(\operatorname{cell}_{G}\left(G / \Gamma_{n} G\right), 1\right)$. However $G / \Gamma_{n} G$ is a $G$-cellular group (see [8, Proposition 7.1(3)]). We conclude $\operatorname{cell}_{X} K\left(G / \Gamma_{n} G, 1\right) \simeq K\left(G / \Gamma_{n} G, 1\right)$, which proves that $K\left(G / \Gamma_{n} G, 1\right)$ is $X$-cellular.

The statement of the next result does not involve cellularity, however we do not know of a proof which does not use this tool. This is the extension to nilpotent fundamental groups of the Bousfield key lemma we presented in the introduction; see also Section 4. It implies for example that, if $X$ is simply connected, then the map $\pi_{n}: \operatorname{map}_{*}(X, X) \rightarrow \operatorname{Hom}\left(\pi_{n} X, \pi_{n} X\right)$ is a weak equivalence if and only if $X$ is weakly equivalent to $K\left(\pi_{n} X, n\right)$. If $X$ is not simply connected, then the situation is much more complicated.

Example 7.7 The $K(\mathbb{Z} / 2,1)$-cellularization of $K\left(\Sigma_{3}, 1\right)$ has already been computed; see [20, Example 2.6]. It is a space $X$ whose fundamental group is the symmetric group $\Sigma_{3}$ and its universal cover is the homotopy fiber of the degree- 3 map on the sphere $S^{3}$. In particular its homotopy groups are non trivial in infinitely many degrees. By the universal property of the cellularization we have weak equivalences of mapping spaces

$$
\operatorname{map}_{*}(X, X) \simeq \operatorname{map}_{*}\left(X, K\left(\Sigma_{3}, 1\right)\right) \simeq \operatorname{Hom}\left(\Sigma_{3}, \Sigma_{3}\right) .
$$

The mapping space of pointed self-maps of $X$ is homotopically discrete, but $X$ is far from being a $K(G, 1)$. This example also shows that the cellularization of nonnilpotent $K(G, 1)$-spaces can become quite complicated.

This is in contrast with what we prove for nilpotent fundamental groups. If the pointed mapping space of self-maps $\operatorname{map}_{*}(X, X)$ is homotopically discrete via the evaluation on the fundamental group, then $X$ must be a $K(G, 1)$. 
Theorem 7.8 Let $X$ be a connected space whose fundamental group $\pi_{1} X$ is nilpotent. Assume that the map $\pi_{1}: \operatorname{map}_{*}(X, X) \rightarrow \operatorname{Hom}\left(\pi_{1} X, \pi_{1} X\right)$ is a weak equivalence. Then $X$ is weakly equivalent to $K\left(\pi_{1} X, 1\right)$.

Proof The assumptions imply that $\operatorname{map}_{*}\left(X, p_{1, X}\right): \operatorname{map}_{*}(X, X) \rightarrow \operatorname{map}_{*}\left(X, P_{1} X\right)$ is a weak equivalence. Thus $X \simeq \operatorname{cell}_{X} K\left(\pi_{1} X, 1\right)$, which by Proposition 7.1, means that $X \simeq K\left(\pi_{1} X, 1\right)$.

Here is another way to restate this result. If the first Postnikov section $X \rightarrow K(G, 1)$ induces a weak equivalence on pointed mapping spaces $\operatorname{map}_{*}(X, X) \simeq \operatorname{map}_{*}(X, K(G, 1))$ and $G$ is nilpotent, then $X$ is a $K(G, 1)$. We also offer a version for higher Postnikov sections. The very same argument as in the proof of Theorem 7.8 can be used to show:

Corollary 7.9 Let $X$ be a connected space whose $n^{\text {th }}$ Postnikov section $P_{n} X$ is nilpotent. Assume that the map $P_{n}: \operatorname{map}_{*}(X, X) \rightarrow \operatorname{map}_{*}\left(P_{n} X, P_{n} X\right)$ is a weak equivalence. Then $p_{n, X}: X \rightarrow P_{n} X$ is a weak equivalence, ie $X$ is an $n-P o s t n i k o v$ stage.

Here is another application of Theorem 6.6 and the characterization of cellularity in Theorem 2.1. Notice however that the statement is not true for a general space $X$. Neither is the analogous statement for higher connected covers of a nilpotent $X$ as we have seen in Example 6.1.

Corollary 7.10 Assume $X$ is nilpotent. If $\operatorname{map}_{*}(X, Y)$ is contractible, then so is $\operatorname{map}_{*}\left(P_{n} X, Y\right)$ for any $n \geq 0$.

As a last application we offer a few results which state simple — but not obvious homological properties of nilpotent spaces. All are immediate consequences of the main theorem above.

Theorem 7.11 Let $\mathcal{K}$ be a reduced homology theory.

(1) Assume $X$ is $\mathcal{K}$-acyclic. If $P_{n} X$ is nilpotent, then it is also $\mathcal{K}$-acyclic and so is $K\left(\pi_{i} X, i\right)$ for $i \leq n$.

(2) Assume $X$ is nilpotent. Then $\prod_{k \geq 1} K\left(\pi_{k} X, k\right)$ is $\mathcal{K}$-acyclic if and only if $\prod_{k \geq 1} K\left(H_{k} X, k\right)$ is $\mathcal{K}$-acyclic.

(3) If $G$ is a group such that $K(G, 1)$ is $\mathcal{K}$-acyclic, then so is $K\left(G / \Gamma_{n} G, 1\right)$ for any $n$. 


\section{References}

[1] M Blomgren, W Chachólski, E D Farjoun, Y Segev, Idempotent transformations of finite groups, Adv. Math. 233 (2013) 56-86 MR2995665

[2] A K Bousfield, Localization and periodicity in unstable homotopy theory, J. Amer. Math. Soc. 7 (1994) 831-873 MR1257059

[3] A K Bousfield, Homotopical localizations of spaces, Amer. J. Math. 119 (1997) 13211354 MR1481817

[4] A K Bousfield, D M Kan, Homotopy limits, completions and localizations, Lecture Notes in Math. 304, Springer, Berlin (1972) MR0365573

[5] W Chachólski, On the functors $C W_{A}$ and $P_{A}$, Duke Math. J. 84 (1996) 599-631 MR1408539

[6] W Chachólski, Desuspending and delooping cellular inequalities, Invent. Math. 129 (1997) 37-62 MR1464865

[7] W Chachólski, A generalization of the triad theorem of Blakers-Massey, Topology 36 (1997) 1381-1400 MR1452856

[8] W Chachólski, E Damian, E D Farjoun, Y Segev, The A-core and A-cover of a group, J. Algebra 321 (2009) 631-666 MR2483285

[9] W Chachólski, W G Dwyer, M Intermont, $v_{*}$-torsion spaces and thick classes, Math. Ann. 336 (2006) 13-26 MR2242617

[10] W Chachólski, P-E Parent, D Stanley, Cellular generators, Proc. Amer. Math. Soc. 132 (2004) 3397-3409 MR2073317

[11] W Chachólski, J Scherer, K Werndli, Homotopy excision and cellularity arXiv: 1408.3252

[12] E B Curtis, Simplicial homotopy theory, Advances in Math. 6 (1971) 107-209 MR0279808

[13] A Dold, R Thom, Quasifaserungen und unendliche symmetrische Produkte, Ann. of Math. 67 (1958) 239-281 MR0097062

[14] W G Dwyer, J P C Greenlees, S Iyengar, Duality in algebra and topology, Adv. Math. 200 (2006) 357-402 MR2200850

[15] E D Farjoun, Cellular inequalities, from: "The Čech centennial”, (M Cenkl, H Miller, editors), Contemp. Math. 181, Amer. Math. Soc. (1995) 159-181 MR1320991

[16] E D Farjoun, Cellular spaces, null spaces and homotopy localization, Lecture Notes in Math. 1622, Springer, Berlin (1996) MR1392221

[17] E D Farjoun, Two completion towers for generalized homology, from: "Une dégustation topologique (Topological morsels): Homotopy theory in the Swiss Alps", (D Arlettaz, K Hess, editors), Contemp. Math. 265, Amer. Math. Soc. (2000) 27-39 MR1803951 
[18] E D Farjoun, R Göbel, Y Segev, Cellular covers of groups, J. Pure Appl. Algebra 208 (2007) 61-76 MR2269828

[19] R J Flores, Nullification and cellularization of classifying spaces of finite groups, Trans. Amer. Math. Soc. 359 (2007) 1791-1816 MR2272149

[20] R J Flores, J Scherer, Cellularization of classifying spaces and fusion properties of finite groups, J. Lond. Math. Soc. 76 (2007) 41-56 MR2351607

[21] T Ganea, A generalization of the homology and homotopy suspension, Comment. Math. Helv. 39 (1965) 295-322 MR0179791

[22] J Kiessling, Classification of certain cellular classes of chain complexes, Israel J. Math. 174 (2009) 179-188 MR2581213

[23] A Libman, Universal spaces for homotopy limits of modules over coaugmented functors, I, Topology 42 (2003) 555-568 MR1953240

[24] H Miller, The Sullivan conjecture on maps from classifying spaces, Ann. of Math. 120 (1984) 39-87 MR750716

[25] J-P Serre, Groupes d'homotopie et classes de groupes abéliens, Ann. of Math. 58 (1953) 258-294 MR0059548

[26] S Shamir, Cellular approximations and the Eilenberg-Moore spectral sequence, Algebr. Geom. Topol. 9 (2009) 1309-1340 MR2520402

Department of Mathematics, KTH Stockholm

Lindstedtsvägen 25, 10044 Stockholm, Sweden

Department of Mathematics, Hebrew University of Jerusalem

Givat Ram, Jerusalem 91904, Israel

Departamento de Matemáticas, Facultad de Ciencias

Universidad Autónoma de Madrid, 28049 Madrid, Spain

Department of Mathematics, EPFL Lausanne

Station 8, 1015 Lausanne, Switzerland

wojtek@math.kth.se, farjoun@math.huji.ac.il, ramon.flores@uam.es, jerome.scherer@epfl.ch

http://gr-he.epfl.ch/Scherer

Proposed: Mark Behrens

Received: 16 January 2014

Seconded: Haynes Miller, Jesper Grodal

Revised: 8 October 2014 